\title{
Personal Emotional Expression in the Creation of Oil Painting
}

\author{
Yuanming Zhang \\ Zhuhai College \\ Jilin University \\ Zhuhai, China
}

\begin{abstract}
Emotion supports and powers oil painting creation, playing a vital role in oil paintings' influence. Oil painting creation never lies in creating the color, space, volume and rhythm. Instead, it lies in painters' subjective processing of these artistic elements to express their own emotion. Emotion, the soul of paintings, powers oil painting creation and runs through the whole process of oil painting creation. We can feel artists' rich emotion before oil painting creation, and it is exactly the emotion which is full of love and humanistic care that impels artists to do oil painting creation. Because this kind of emotion nourishes artists' soul, builds up their aesthetic perception, becomes the truest part of the soul with richest emotion, and can inspire artists' expression enthusiasm and creation desire to the utmost. Colors in oil painting creation are subjective and derive from combination of personal emotion and objective colors instead of reproducing true colors of objective things.
\end{abstract}

Keywords—oil painting creation; personal emotion; emotional expression

\section{INTRODUCTION}

The charm of oil painting lies in true feelings it conveys rather than its level of similarity with reality by imitation. Especially in nowadays, if you only pursue being life-like and real, you can use photos to express your emotion, speedy and simple. In this case, there is no need of oil painting creation requiring creators to rack their brains. The process of oil painting creation is also the process of artists' personal emotional expression and a carrier to express emotions, depicting artists' inner true feelings. We regard it as a way to evaluate oil paintings. Therefore, emotion supports and powers oil painting creation, playing a vital role in oil paintings' influence.

\section{THE IMPORTANT ROLE OF EMOTION IN OIL PAINTING CREATION PROCESS}

Emotion is the motive force and source of artistic creation. Human's emotion is invisible, untouchable and intangible, and it can only be expressed by mediums. As we all know, art originates from life. Oil painting is an important part of art and an important medium for emotional expression. Creator's personal emotion in oil painting is the key to achieve excellence, move viewers and connect with them. Only when a painting can touch its own creator, can it possibly touch other people. Only personal emotion can make oil paintings attractive and even can endow it with personality charm. Oil painting creation never lies in creating the color, space, volume and rhythm. Instead, it lies in painters' subjective processing of these artistic elements to express their own emotion. Emotion, the soul of paintings, powers oil painting creation and runs through the whole process of oil painting creation.

\section{DIFFERENT PHASES OF EMOTIONAL EXPRESSION IN OIL PAINTING CREATION PROCESS}

\section{A. Personal Emotion Motivates Forming of Creative Impulse}

Artistic creation originates from artists' artistic creation impulse, whose formation process is also the process of emotional flooding's generation and rational release. Pure sensory stimulation and subjective wishes can't generate creation impulse, the formation of which is sustained in artists' acute sense, rich social and emotional experiences and highlevel professional knowledge. Generally speaking, creation impulse is an inevitable mental result due to appearance of accidental phenomena and an epiphanic emotional response originating from recovery and enhancement of aesthetic experience. A certain kind of people, things, objects and views can trigger associations with kindness, evil, beauty and ugliness in people's mind, typified and artistic processing of which generates creation impulse. Kindness, evil, beauty and ugliness, the most real and clearest part of people's emotion, reflect creators' values and determine generation of creation impulse. Observation, experience and feeling are the bridge through which emotion leads to creation impulse. Xin Dongwang, a renowned oil painter in China, makes great efforts to observe and think about the model and scene to achieve the strongest creation impulse before creating each painting. Among his numerous character works, Beauty is an ordinary one, displaying a full composition of a middle-aged countryside woman. In the voiceover, $\mathrm{Mr}$ Xin Dongwang writes as follows: "Always we are excited in brewing a new painting at the beginning of a week. However, the assigned model in teaching aids room is beyond all of your expectations. No matter what, we can't show any expression that might hurt the model's feeling. I gradually feel her mood in our conversation and artistic inspiration comes into being. It is destined to be an imperfect work, thus it is more worthy to capture her inner conflict than to produce a beautiful painting of her. She is entranced and reserved due to her inner yearn for beauty. After a whole day's repeated arrangements, I finally 
decide she wears a braces skirt. In the moment of starting rough sketch, I suddenly feel plainness and elegance, like those of figurines in Han Dynasty and sculptures in Song Dynasty, in her look." 1 From it, we can feel artist's rich emotion before oil painting creation. It is exactly the emotion which is full of love and humanistic care that impels generation of oil painting creation impulse.

\section{B. Emotion Influences the Theme Selection of Oil Painting Creation}

Oil painting's theme is expression of emotion towards things and originates from daily life instead of creation without foundation. Therefore, oil painting's theme lies in the content which is associated with oil painting creators, influences creators, conforms to creators' feelings and touches creators. Only the above mentioned theme can reflect creators' real feelings, express creators' inner desire and arouse people's resonance. For example, when appreciating famous paintings, we can refer to works' themes to infer creators' living environment and historical background and deeply feel the emotion and content they strive to convey.

During the process of theme selection, artists artistically process the things they see, hear and imagine and choose something which can mostly express their previous creation impulse. Theme selection depends on artists' depth of thought, aesthetic taste and artistic culture and artists usually select themes from their life practice, growth environment and spirit world. Because, as the most real and emotional part of mind, these things nourish artists' mind, establish their aesthetic conception and can mostly inspire artists' expressive enthusiasm and creative desire. In his short life, Vincent Van Gogh bears a solitary character and is bad at communicating with others. As a result, he becomes interested in real and beautiful plants or scenery around him and can use his favorite way to execute his subjective judgment on object modeling or color in painting them. He is quite happy because there is no others' supercilious look and evil suspicion in the process. Therefore, most of Van Gogh's painting themes are sunflowers, flowers, plants, himself and surrounding scenery. Miller's The Gleaners depicts the scene that three women bend their bodies and pick up ears of wheat in the field carefully to feed their family after autumn harvest. They are in coarse cloth costume and cumbersome shoes with plump figure. They are barely beautiful, let alone elegant. Grown up in a pheasant family, Miller bears a deep memory of and deep emotion towards labor scenes in Barbizon fields. Miller uses warm yellow in the painting, making the whole painting solemn and tranquil and expressing Miller's love for countryside life and sympathy for peasant women in a good way. Even in nowadays, Miller's oil paintings with peasant theme are praised, because his works enhance scenes with full emotion to the level of labor beauty which is a kind of praise and eulogizing. These works arouse common feelings among appreciators and bring them to a heavenly-like realm, thus transforming emotion into a kind of spiritual enjoyment.

\footnotetext{
${ }^{1}$ Xin Dongwang. Particulars . Xin Dongwang[M]. People's Fine Arts Publishing House. 2011, 01:126.
}

\section{Emotion Influences the Process of Oil Painting Creation}

Oil painting creation is a process of sparing no efforts and it needs painter's tremendous efforts in selection and collection of oil painting theme, determination of oil painting form and combination with personal life experience and many other elements to finally accomplish oil painting works. The whole process of creation is accompanied by continuous release of emotion and contains all of painter's emotion and their countless painstaking care and inspiration. Completion of creation isn't accomplishment of the whole work. Painter's constant improvement and perfection are needed. Painter's cognition and emotion towards the painting get enhanced in late improvement process and the improved work is perfect.

\section{Form of Emotional Expression in Oil Painting Creation}

1) Line: One major part of oil painting is line, which directly constitutes the entire oil painting. In real life, we can't feel lines intuitively. Line, a product of human's abstract thinking, manifests human's subjective initiative. Humans create various categories of lines through subjective imagination and direct feelings about real life. On this basis, it becomes a popular visual feature to perceive and know about surrounding things through lines. There are various forms of lines, like empty and full, curved and straight, thick and thin, and long and short. People have different feelings about different lines. For example, straight lines represent upright, tall and straight; curved lines represent lively and graceful; horizontal line sustains the peaceful and broad feeling of roaring waves, bringing random thoughts to people's brain as if they saw the real horizontal line and sea level. Besides, various directions of lines bring different feelings to people. Lines varying in width, depth and length also express different emotion. Lines originate from the painter's soul and reflect vigorous artistic ecology running through the whole painting creation process. In the Hypnagogic Yunus, one of Giorgione's oil painting works, Yunus is in sound sleep among natural scenery with a gentle, elegant, undulating, easy-going and well-proportioned body, displaying no religious features and becoming integrated with the nature. This kind of elegant and beautiful line is to demonstrate the unity of human's pure beauty in mind and vitality instead of giving people sensual stimulus. This kind of sense of beauty which is full of humanism corresponds to requirements for beauty in renaissance.

Lines also have decorative effects. For example, one unique decoration method of Black Hair Nude Girl(Schiller, 1911 ) is drawing body with various lines, combining disgust at and attraction of erotica. Another example is Dry Laundry (Schiller, 1917), in which the painter uses angular lines to demonstrate soft clouds in the sky and textured clothes. Smooth curved lines outline clothes in various styles which float with the wind, displaying a strong decorative sense together with clouds over faraway mountains and wellproportioned houses. Schiller's various kinds of paintings in different periods demonstrate his painting features like unique colors, bold and indulged lines and exaggerated modeling. Schiller demonstrates images in an exaggerated way through jerky brushwork, cold and broken color, and most changeful lines. 
Schiller also has a great insight into modeling design. For example, figures under Schiller's brushwork have a succinct modeling and a contractive, slim and exaggerated body with a strong undulating feeling which are outlined through straight lines; or transform the figure's costume and use exaggerated colors and patterns in painting to create a strong comparison effect. Schiller's unique artistic style can't do without this kind of subjectively willful means of expression. For example, in the Self-portrait, a painting work of Schiller in 1910, character modeling is extremely exaggerated with bent and disarticulated body, stiff fingers and twisted upper limbs of the painter. There are no redundant curves and flesh and blood in the painter's body, leaving behind textured muscle, physique, lines and geometric structure with distinctive edges, as if the painter was pouring out dissatisfaction towards reality and inner hesitation and gloom. In terms of line application, Schiller's talent is higher than his teacher, Gustav Klimt. Gustav Klimt is good at depicting women's soft body through smooth lines. Very different from his teacher in expressing method in figure drawing, Schiller prefers application of subjective imagination to stress skinny body and present rise and fall in human body, which can be called capturing body form in prevailing deconstructivism thought at that time. For example, in the Embrace (Schiller, 1917), application of line is perfect. The two lovers lie there, embracing each other. Dynamic composition of figure and smooth lines highlight figures' elegant and beautiful geometric bodies. Colors in strong comparison, and textured lines create an amazingly strong and weird scene, reflecting people's emotion of painful struggle under the cover of consciousness of Judgment Day on the previous day of World War I. Schiller's extremely imaginative decorative method contributes a lot to composition and lines.

2) Color: Color is an extremely important painting language in oil painting creation. Color in oil painting creation is not a reproduction of object things' real color. Instead, it is a subjective color formed by combination of personal emotion and objective color. Application of subjective color can create the kind of message and effect which can not be conveyed through body language. Subjective color is the most appropriate color selected by the painter's aesthetic emotion inspired by drawing object under the circumstance which conforms to the painter's color sensation. So to speak, subjective color is emotional color. Oil painting creator uses color changes to demonstrate changes in personal mood and emotion and different colors have different meanings. For example, red color represents impetus, vigor, excitement and passion, orange represents creation, dependency and connection; yellow color represents control desire, willpower, confidence, self-assertiveness and authority; green color represents harmony, sense of space, mildness and energy; blue color has strong sensitiveness and great insights into the whole thing, representing perception; purple color represents intelligence, mystery, nobleness and wisdom. Painters use these colors to endow their expressive space with infinite possibilities and appreciators can directly communicate with painting works through changes of color.

For example, in the Dance, Mattis not only uses oriental brush drawing techniques, but also uses music, dance and other different artistic elements to express his own painting creation language. Mattis paints the Dance from 1909 to 1910. During the painting process, Mattis goes to Mediterranean shore together with the model. The joviality Mediterranean brings to him is closely related to the emotion the whole painting desires to express. The painting adopts blue as its background color, referring to the blue sky of southern coast in August. Green background makes people involuntarily think of green grassland. The Mediterranean people's tan healthy body is depicted incisively and vividly through vermilion. In this bold and unrestrained wilderness painting, dancers ceaselessly twist their bodies, hold hands to make a circle and crazily wave their arms and legs, as if they were kidnapped by a kind of primitive and robust rhythm.

\section{CONCLUSION}

Emotionless oil paintings are tasteless and hypodynamic and even valueless. The previously emotionless natural scenery becomes attractive because of injection of emotion and the most ordinary laborers are extremely charming because of existence of emotion. The process of oil painting creation is about expression of artists' inspiration from life and growth feeling, through which artists convey their emotion to other people and communicate with others. The content of oil painting creation comes from real life, acting as a kind of personal emotion expression for the painter. In order to create an oil painting recognized by everyone, the painter needs to start from daily life to look for the appropriate painting theme and put personal emotion into painting. Excellent oil painting works don't need painter's vivid depiction of reality. Instead, it needs emotion and connotation to arouse resonance among the audience. Due to the existence of emotion, excellent oil paintings have infinite infectivity, providing a spiritual homeland for artists and spiritual baptism for appreciators.

\section{REFERENCES}

[1] Bi Wenjiao. Analysis on Personal Emotion Expression in Oil Painting Creation[D]. Shandong Normal University, 2015, 06:213-216.

[2] Du Xiaohui. Analysis on Emotional Elements in Oil Painting Creation[J]. Popular Literature, 2016,04: 86-87.

[3] Li Bing. Concentrated Demonstration of Personal Emotion in Oil Painting Creation[J]. Modern Decoration(Theory), 2014,09:158.

[4] Zhong Pei. Analysis on Emotional Expression in Oil Painting Creation[J]. Art Appreciation, 2015,03:196.

[5] Zhu Qianru. Analysis on Emotional Expression in Oil Painting Creation[D]. Northwest Normal University, 2016,05:22. 